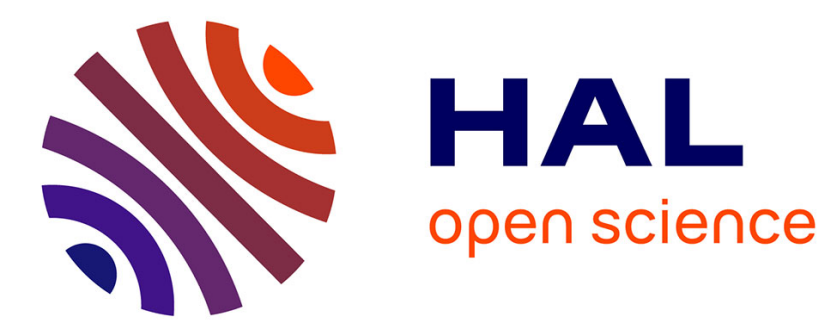

\title{
Inhibitory control of spike timing precision
}

Maxime Ambard, Dominique Martinez

\section{To cite this version:}

Maxime Ambard, Dominique Martinez. Inhibitory control of spike timing precision. Neurocomputing, 2006, 70 (1-3), pp.200-205. 10.1016/j.neucom.2006.03.010 . inria-00401772

\section{HAL Id: inria-00401772 \\ https://hal.inria.fr/inria-00401772}

Submitted on 6 Jul 2009

HAL is a multi-disciplinary open access archive for the deposit and dissemination of scientific research documents, whether they are published or not. The documents may come from teaching and research institutions in France or abroad, or from public or private research centers.
L'archive ouverte pluridisciplinaire HAL, est destinée au dépôt et à la diffusion de documents scientifiques de niveau recherche, publiés ou non, émanant des établissements d'enseignement et de recherche français ou étrangers, des laboratoires publics ou privés. 


\title{
Inhibitory control of spike timing precision
}

\author{
Maxime Ambard and Dominique Martinez \\ CORTEX Group, LORIA-INRIA, Nancy, France \\ ambard@loria.fr,dmartine@loria.fr
}

\begin{abstract}
GABAergic inhibition via local interneurons may play a role in enhancing spike timing precision in principal cells, since it tends to eliminate the influence of initial conditions. However, both the number and the timing of inhibitory synaptic events may be variable across repeated trials. How does this variability affect the spike timing precision in principal neurons? In this paper, we derive an analytical expression for the spike output jitter as a function of the variability of the received inhibition. This study predicts that variable inhibition is especially tolerated as the number of inhibitory cells is large, which is consistent with experimental data from early olfactory systems (antennal lobe for insects, olfactory bulb for vertebrates).
\end{abstract}

\section{Introduction}

Experimental evidence tends to show that precise spike timing plays a significant role in the encoding of sensory stimuli [1]. A pre-requisite is that neurons fire spikes in a precise and reproducible way over repeated presentations of the same stimulus. Both experimental studies and theoretical works have shown that the neural response can indeed be precise and reliable, depending on the nature of the input $[2,3,4]$. Fast varying aperiodic stimuli lead to precise spike timing while constant or slowly varying stimuli yield imprecise firing. All natural stimuli however do not have a high-temporal bandwidth. For example, in comparison with sounds or images, odors change more slowly.

Olfaction is generally a slow-temporal bandwidth sense. Olfactory receptor neurons converge onto glomeruli that present a slow dynamics of activation [5]. Thus, olfactory bulb mitral cells (MCs), excited by one or few glomeruli, receive slowly varying inputs. It is known that MCs have an unreliable spiking activity under constant stimulation [6]. Despite this fact, some MCs present in vivo synchronisation with precise spiking activity $[7,8]$. In the olfactory bulb, MCs receives inhibition from inhibitory granule cells (GCs). The received inhibition could be responsible of the precision of individual MCs, since it tends to eliminate the influence of initial conditions $[9,10]$. However, GABA release from GCs is random [11], and thus inhibitory feedback into the MCs is a stochastic process. How does the stochastic nature of the received inhibition affect the precision of principal cells ? To address this 
question we shall use a quadratic integrate-and-fire neuron model that allows for analytic calculations. In section 2, we describe our model and present simulations showing that GABAergic inhibition may enhance spike timing precision. In section 3 , we derive an approximate analytical expression for the spike output jitter as a function of the variability of the received inhibition. In section 4 , we demonstrate the validity of this approximation with simulation results. In section 5 , we discuss the predictions obtained from our study.

\section{Model description and Simulations}

We consider here the quadratic integrate and fire (QIF) model which is known to be a very good approximation of any type 1 neuron [12]. The time evolution of the membrane potential $V$ is described by the following equation

$$
C \frac{d V}{d t}=q\left(V(t)-V_{T}\right)^{2}+I-I_{t h}-I_{G A B A}(t)+I_{\text {noise }}(t)
$$

in which $I$ is a constant input current, $I_{t h}$ denotes the minimal current required for repetitive firing, $I_{\text {noise }}$ is an intrinsic white noise current of standard deviation $\sigma_{\text {noise }}$ and $I_{G A B A}$ is a synaptic inhibitory current. In the absence of any noise and synaptic current, the QIF neuron converges to the resting potential $V_{\text {rest }}$ when $I=0$ and fires as soon as $V$ reaches the threshold $V_{t h}$, when $I \geq I_{t h}$. Right after the spike, $V$ is reset to the value $V_{\text {reset }}$. The membrane capacitance $C$ and resting potential $V_{\text {rest }}$ have been derived from MC experimental data [13] [14]. The other parameters have been fitted in order to obtain a similar frequency-current response than the $\mathrm{MC}$ conductance based model in [15]. The parameters chosen for the QIF neuron are as follows : $C=0.2 \mathrm{nF}, V_{\text {rest }}=-65 \mathrm{mV}, V_{T}=-60.68 \mathrm{mV}$, $q=0.00643 \mathrm{mS} . V^{-1}, I_{t h}=0.12 \mathrm{nA}, V_{t h}=30 \mathrm{mV}$ and $V_{\text {reset }}=-70 \mathrm{mv}$.

The inhibitory synaptic current $I_{G A B A}(t)$ in Eq. 1 results from the summation of GABAergic synaptic events originating from interactions with GCs. An unitary event, occuring at time $t^{f}$, is modeled by a simple exponential inhibitory postsynaptic current (IPSC). For $t \geq t^{f}$, we have

$$
\operatorname{IPSC}(t)=g e^{-\left(t-t^{f}\right) / \tau}\left(V(t)-E_{G A B A}\right)
$$

The maximum synaptic conductance is $g=1 \mathrm{nS}$ [14], the synaptic time decay is $\tau=6 \mathrm{~ms}[16]$ and the reversal potential of the synapse is $E_{G A B A}=-70 \mathrm{mV}$.

The QIF neuron is precise when its firing time $T$ stays unchanged across repeated trials with the same input current $I$. A measure of precision is the spike time jitter $\sigma_{T}$ which characterizes the temporal dispersion around cluster firing times induced by repeated trials (precise neuron $=$ small $\sigma_{T}$ ). Figure 1 shows the temporal evolution of $\sigma_{T}$, estimated over 100 repeated simulations of the QIF neuron (Eq. $1)$, with $I_{G A B A}=0$ and different $\sigma_{\text {noise }}$ values. The initial condition $V(t=0)$ was similar in all trials. Due to $I_{\text {noise }}$, the spike time jitter $\sigma_{T}$ increases over time so that the neuron becomes more and more imprecise. This is in agreement with previous works $[2,3,4]$. To see if the first spike can convey some information about the input, we performed repeated simulations for different $I$ and random initial conditions. Figure 2 shows the mean and standard deviation $\sigma_{T}$ of the first spike latency for a QIF neuron, with and without GABAergic inhibition. When $I_{G A B A} \neq 0,100$ synchronous IPSCs are generated at time $t^{f}=0$ according to Eq. 2. From Fig. 2, we see that $\sigma_{T}$ is very large when $I_{G A B A}=0$ and very small when $I_{G A B A} \neq 0$. GABAergic inhibition makes the neuron more precise so that its firing 
time $T$ is a reliable estimate of $I$. Two examples of spike rasters are indicated in Fig. 2 for $I=0.14 n A$ and for $I_{G A B A}=0$ and $I_{G A B A} \neq 0$.

In our simulations, GABAergic inhibition tends to eliminate the influence of initial conditions, in line with previous works $[9,10]$. In the olfactory bulb, the received inhibition from GCs could therefore be responsible for the precision of individual MCs. However, GABA release from GCs is random [11] and thus inhibitory feedback into the MCs will be variable across trials. How does the variability of the received inhibition affect the precision of principal neurons ? This point will be studied mathematically in the next section.

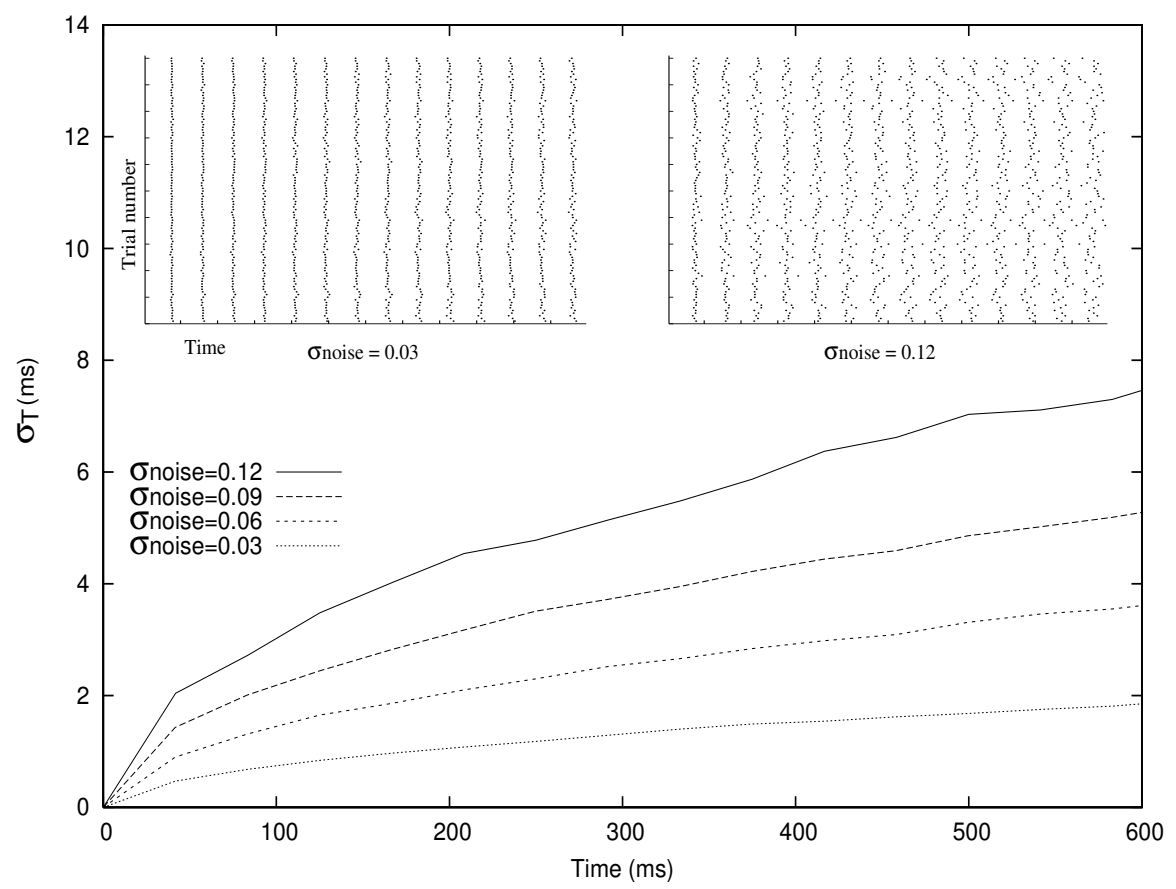

Figure 1: Without GABAergic inhibition, the spike time jitter $\sigma_{T}$ increases over time. The different curves indicate the temporal evolution of the spike time jitter $\sigma_{T}$ estimated over 100 repeated simulations of the QIF neuron (Eq. 1). In the simulations, $I=0.15 n A, I_{G A B A}=0$ and $\sigma_{\text {noise }}$ ranges from 0.03 to $0.12 n A$. Two examples of spike rasters obtained from 100 trials are indicated for $\sigma_{\text {noise }}=0.03$ and $0.12 n A$.

\section{Mathematical analysis of spike timing precision for a neuron receiving variable inhibition}

Let us first consider a QIF neuron (Eq. 1 with $I_{\text {noise }}(t)=0$ ) receiving, at a time $t^{f}$, a single IPSC whose temporal evolution is given by Eq. 2. The total current, for $t \geq t^{f}$, is then

$$
J(t)=I-I_{t h}-g e^{-\left(t-t^{f}\right) / \tau}\left(V(t)-E_{G A B A}\right)
$$

Börgers and Kopell [9] have shown that the firing time $T_{1}$ of a QIF neuron receiving a single strong IPSC is relatively independent of the initial condition $V(t=0)$, see 


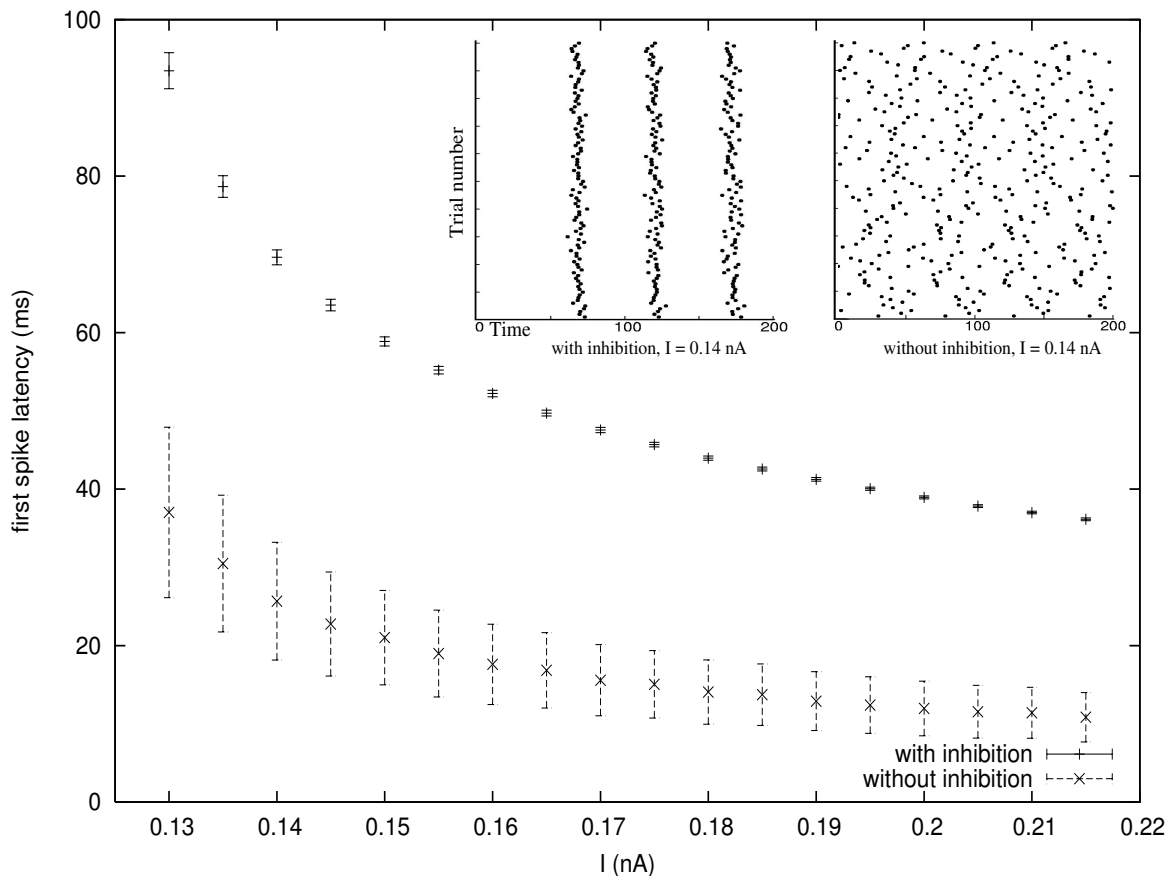

Figure 2: Synchronous GABAergic inhibition enhances spike timing precision. Mean and standard deviation of the first spike latency as a function of the input current $I$ have been estimated over 100 repeated simulations of the QIF neuron (Eq. 1, $\sigma_{\text {noise }}=0.05 n A$ ). The top curve is for $I_{G A B A} \neq 0$ whereas the one at the bottom is for $I_{G A B A}=0 . I_{G A B A} \neq 0$ was obtained by the summation of 100 synchronous synaptic events occuring at time $t^{f}=0$ and modeled by Eq. 2 . Two spike rasters, obtained from repeated trials with $I=0.14 n A$, are indicated for $I_{G A B A}=0$ and $I_{G A B A} \neq 0$.

also [10]. Provided $g$ is large enough, trajectories in the phase plane $(V, J)$ are all attracted towards a given trajectory so that they all reach approximately the same point $\left(V_{t h}, J^{\star}\right)$ at firing time. This is shown in Fig. 3 in [9] and Fig. 5C in [10]. The result is an almost complete loss of the initial condition $V(t=0)$. Whatever the initial condition might be, the total input current is approximately equal to $J^{\star}$ at the firing time $T_{1}$

$$
J^{\star} \approx J\left(T_{1}\right)=I-I_{t h}-g e^{-\left(T_{1}-t^{f}\right) / \tau}\left(V_{t h}-E_{G A B A}\right)
$$

and thus

$$
T_{1} \approx \tau \ln g-\tau \ln \left(I-I_{t h}-J^{\star}\right)+\tau \ln \left(V_{t h}-E_{G A B A}\right)+t^{f}
$$

To determine the effect of variable inhibition on the spike time jitter, we have generalized Börgers and Kopell's study to the case of a QIF neuron receiving a burst of $k$ IPSCs at times $t_{i}^{f}, i=1,2 \cdots k$. Without loss of generality, we consider that the neuron fires after receiving the $k$ th IPSC. At the firing time $T$, the total 
input current is approximately equal to $J^{\star}$

$$
J^{\star} \approx J(T)=I-I_{t h}-\sum_{i=1}^{k} g e^{-\left(T-t_{i}^{f}\right) / \tau}\left(V_{t h}-E_{G A B A}\right)
$$

and thus, the firing time of a neuron receiving a burst of $k$ IPSCs is

$$
T \approx \tau \ln g-\tau \ln \left(I-I_{t h}-J^{\star}\right)+\tau \ln \left(V_{t h}-E_{G A B A}\right)+\tau \ln \sum_{i=1}^{k} e^{t_{i}^{f} / \tau}
$$

Let us now consider variable inhibition, i.e. the number $k$ of received IPSCs is a random variable with mean $\langle k\rangle$ and variance $\sigma_{k}^{2}$, and the IPSC times $t_{i}^{f}$ are drawn randomly from an unknown probability density function with variance $\sigma_{t}^{2}$. The only random variable in Eq. (4) is thus

$$
X=\tau \ln \sum_{i=1}^{k} e^{t_{i}^{f} / \tau}
$$

Furthermore, we have $\sigma_{T}^{2}=\sigma_{X}^{2}$. An approximation for $\sigma_{T}^{2}$ can be found by considering the fact that the variance of a sum of a random number $k$ of independent random variables, each with variance $\sigma^{2}$, is $\langle k\rangle \sigma^{2}+\langle k\rangle^{2} \sigma_{k}^{2}$, and that the variance of a function $y=g(x)$ of a random variable $x$ approximately depends on the mean $\eta_{x}$ and variance $\sigma_{x}^{2}$ of $x: \sigma_{y}^{2} \approx|d g / d x|_{x=\eta}^{2} \sigma_{x}^{2}$ (eq. 5-56 in [17]). Using these formulae, we found

$$
\sigma_{T}^{2} \approx \frac{1}{<k>}\left(\sigma_{t}^{2}+\tau^{2} \frac{\sigma_{k}^{2}}{<k>}\right)
$$

Note that Eq. 5 becomes identical to Eq. (4.20) in [9] when the inhibition is precise $\left(\sigma_{t}^{2}=0\right)$. However, Eq. 5 is more general than the one in [9] because it takes into account the fact that the IPSCs can occur at different times.

\section{Numerical results}

In order to check the validity of the approximation given by Eq. 5, we performed repeated simulations of the QIF neuron (Eq. 1, $\left.I_{\text {noise }}(t)=0\right)$ receiving a burst of $k$ stochastic asynchronous synaptic events (Eq. 2). The number $k$ of unitary IPSCs is drawn randomly from a gaussian density with mean $<k>=100$ and standard deviation $\sigma_{k}$ varying from 0 to 9 . Unitary IPSCs are generated at random times (inhibitory jitter $\sigma_{t}$ taken from 0 to $9 \mathrm{~ms}$ ). Figure 3 compares the theoretical spike time jitter $\sigma_{T}$ given by Eq. 5 to the one obtained from simulations. When the inhibition is precise (small $\left.\sigma_{t}\right)$ and balanced $\left(k \approx<k>\right.$, small $\left.\sigma_{k}\right)$, we see a perfect match between theoretical and experimental $\sigma_{T}$ values. For $\sigma_{t}$ larger than $4 \mathrm{~ms}$, $\sigma_{T}$ values given by Eq. 5 are however underestimated. Moreover, the discrepancy between theoretical and experimental $\sigma_{T}$ values increases with the inhibitory jitter $\sigma_{t}$. This is due to the approximations made for deriving Eq. 5. In particular, the approximation of the variance of a function (eq. 5-56 in [17]) is only valid when the variance is small. From Eq. 5, we see that the inhibitory jitter contributes negatively

to the spike timing precision through the ratio $\sigma_{t}^{2} /<k>$. Because the mean number 
of IPSCs was large in our simulations, we obtained $\sigma_{T}<<\sigma_{t}$ (see Fig. 3). So far, we just considered a single burst of inhibition with $\langle k\rangle$ fairly large. What happens to the spike time jitter when $\langle k\rangle$ is smaller and the inhibition phasic? To adress this question, we performed simulations of a QIF neuron receiving consecutive bursts of stochastic asynchronous synaptic events. Figure 4 shows the temporal evolution of $\sigma_{T}$ after consecutive bursts of inhibition. We see that $\sigma_{T}$ reaches a stable state that does not depend on initial conditions but does depend on the value of $\sigma_{k}$.

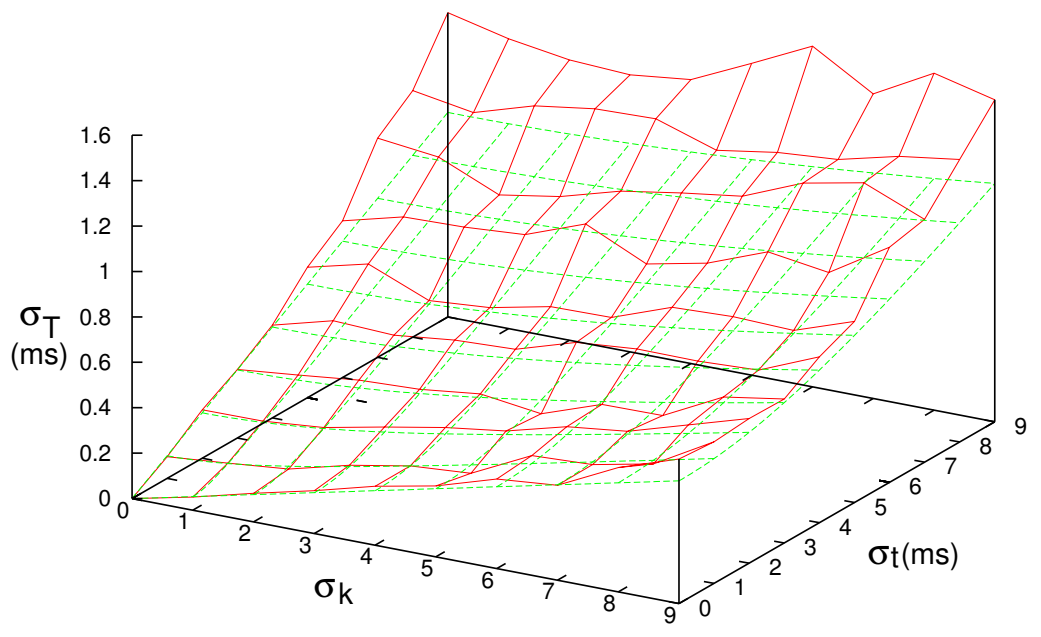

Figure 3: Spike time jitter $\sigma_{T}$ as a function of the variability of the received inhibition $\left(\sigma_{t}, \sigma_{k}\right)$. The dashed curve represents theoretical $\sigma_{T}$ values given by Eq. 5 . The plain curve represents experimental $\sigma_{T}$ values obtained from simulations of the QIF neuron. In Eq. $1, I_{\text {noise }}(t)=0, I=0.13 n A$ and $I_{G A B A}$ was obtained from a burst of $k$ stochastic asynchronous synaptic events (Eq. 2). The number $k$ of unitary IPSCs is drawn randomly from a gaussian density with mean $\langle k>=100$ and standard deviation $\sigma_{k}$ varying from 0 to 9 . Unitary IPSCs are generated at random times (inhibitory jitter $\sigma_{t}$ taken from 0 to $9 \mathrm{~ms}$ ).

\section{Discussion}

We have considered the spike timing precision of a neuron receiving GABAergic inhibition. Due to the stochastic nature of the inhibition, the number and the timing of inhibitory synaptic events is variable across repeated trials. How does this variability affect the spike timing precision in principal neurons ? We have derived an approximate analytical expression for the spike output jitter (Eq. 5). The variability of the received inhibition is characterized by the inhibitory jitter $\sigma_{t}^{2}$ and the variance $\sigma_{k}^{2}$ in the number $k$ of inhibitory events. The inhibition is said to be balanced when $\sigma_{k}^{2}$ is small so that $k$ across trials is approximately equal to the mean inhibition $\left\langle k>\right.$. The inhibition is said to be precise when $\sigma_{t}^{2}$ is small so that the inhibitory events occur approximately at the same time. From Eq. 5, we see 


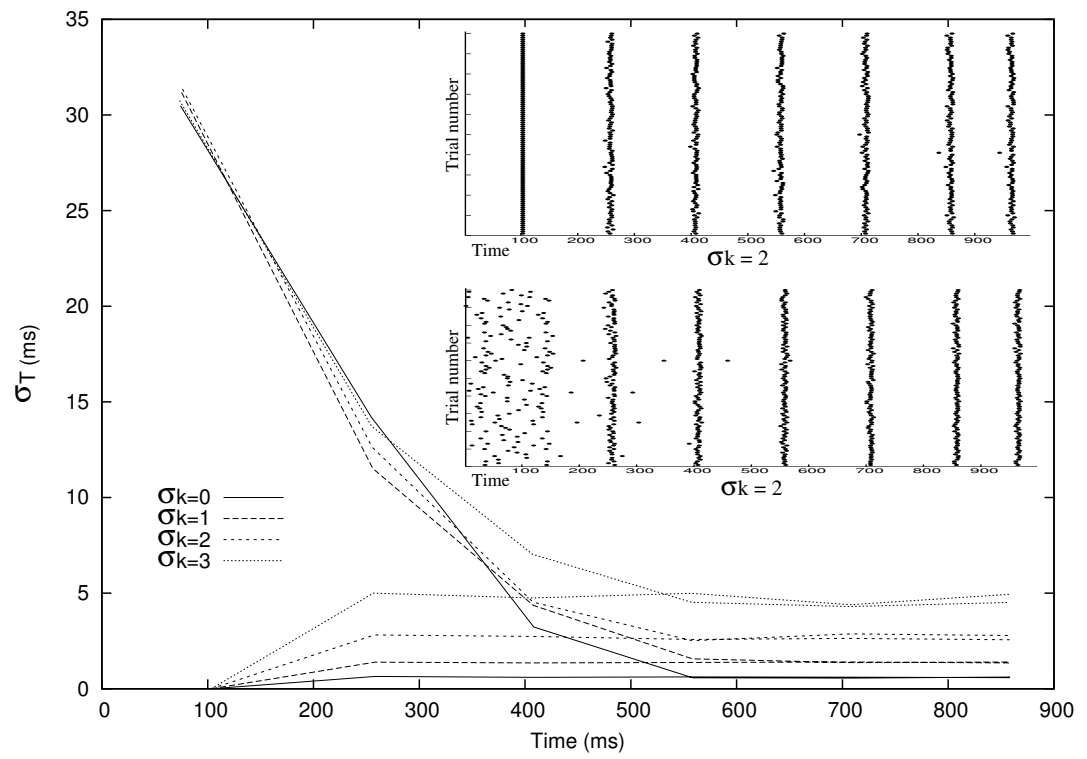

Figure 4: With phasic GABAergic inhibition, the temporal evolution of the spike time jitter $\sigma_{T}$ reaches a stable state. The QIF neuron (Eq. 1, $\left.I_{\text {noise }}(t)=0\right)$ receives consecutive bursts of stochastic asynchronous synaptic events $\left(<k>=10\right.$ IPSCs, $\sigma_{t}=2 \mathrm{~ms}, \sigma_{k}$ varying from 0 to 3 IPSCs, period of inhibitory bursts $=150 \mathrm{~ms})$. Two extreme initial conditions are considered, one for which $V(t=0)$ was similar in all trials and another for which $V(t=0)$ was randomly chosen. They respectively lead to $\sigma_{T}=0 \mathrm{~ms}$ and $\sigma_{T} \approx 30 \mathrm{~ms}$ at time $t=100 \mathrm{~ms}$. Despite different initial conditions, $\sigma_{T}$ converges to a stable state which depends on the value of $\sigma_{k}$. Two spike rasters, obtained from repeated trials with $I=0.125 n A$, are indicated for the two initial conditions.

that the contribution of $\sigma_{t}^{2}$ and $\sigma_{k}^{2}$ to the spike output jitter $\sigma_{T}^{2}$ is divided by the mean inhibition $\left\langle k>\left(\right.\right.$ large $<k>$ implies small $\left.\sigma_{T}^{2}\right)$.

In neural structures with a large number of inhibitory cells, $\langle k\rangle$ is expected to be large, and thus there is no requirement to have precise and balanced inhibition. In contrast, precise spike timing in neural structures where $\langle k\rangle$ is small requires precise and balanced inhibition. This prediction is in line with previous work [18]. It is also in agreement with experimental data from early olfactory systems (antennal lobe for insects, olfactory bulb for vertebrates). On the one hand, the olfactory bulb has a large number of inhibitory cells (e.g. $\sim 10^{6}$ for the mouse) and the inhibition is not precise $\left(\sigma_{t} \approx 22 \mathrm{~ms}\right.$, see Fig. 4B2 in [16]). On the other hand, the antennal lobe has a small number of inhibitory cells (e.g. 300 for the locust) and the inhibition is very precise $\left(\sigma_{t} \approx 3.8 \mathrm{~ms}\right.$, see [19]).

Other lines of research as extensions to this work are interesting to pursued, in particular the role of phasic inhibition. As seen in Fig. 4, the firing times of a neuron receiving consecutive bursts of inhibition become more and more precise over time. On the contrary, the spike time jitter of a residual neuron that does not receive inhibition increases over time so that only its firing rate can reliably encode information (see Fig. 1). This suggests that complementary pieces of information may be conveyed in the precise timing of inhibited neurons and in the firing rate 
of residual neurons. Phasic inhibition could therefore multiplex information into separate channels, in agreement with recent experimental work [8].

\section{References}

[1] R. VanRullen, R. Guyonneau, and S. J. Thorpe. Spike times make sense. Trends in Neurosciences, 28:1-4, 2005.

[2] Z. Mainen and T. Sejnowski. Reliability of spike timing in neocortical neurons. Science, 268:1503-1506, 1995.

[3] R. Brette and E. Guigon. Reliability of spike timing is a general property of spiking model neurons. Neural Computation, 15:279-308, 2002.

[4] B. Gutkin, G. B. Ermentrout, and M. Rudolph. Spike generating dynamics and the conditions for spike-time precision in cortical neurons. J. Comput. Neuroscience, 15:91-103, 2003.

[5] S. Sachse and C. G. Galizia. The coding of odour-intensity in the honeybee antennal lobe: local computation optimizes odour representation. Eur. J. Neurosci., 18:21192132, 2003.

[6] R. Balu, P. Larimer, and B. W. Strowbridge. Phasic stimuli evoke precisely timed in intermittently discharging mitral cells. J. Neurophysiol, 92:743-753, august 2004.

[7] H. Kashiwadani, Y. F. Sasaki, N. Uchida, and K. Mori. Synchronized oscillatory discharges of mitral/tufted cells with different molecular receptive ranges in the rabbit olfactory bulb. Journal of Neurophysiology, 82:1786-1792, 1999.

[8] R. W. Friedrich, C. J Habermann, and G. Laurent. Multiplexing using synchrony in the zebrafish olfactory bulb. Nature Neuroscience, 7:862-871, 2004.

[9] C. Börgers and N. Kopell. Synchronisation in network of excitatory and inhibitory neurons with sparse, random connectivity. Neural Computation, 15:509-538, 2003.

[10] N. Kopell and B. Ermentrout. Chemical and electrical synapses perform complementary roles in synchronization of interneuronal networks. PNAS, 101:15482-15487, 2004.

[11] V. Egger, K. Svoboda, and Z. F. Mainen. Dendrodendritic synaptic signals in olfactory bulb granule cells: local spine boost and global low-threshold spike. The journal of neuroscience, 25:3521-3530, 2005.

[12] B. Ermentrout. Type 1 membranes, phase resetting curves, and synchrony. Neural computation, 8:979-1001, 1996.

[13] G. Lowe. Inhibition of backpropagating action potential in mitral cell secondary dendrites. J. Neurophysiol., 88:64-85, 2002.

[14] Z. Nusser. Release-independent short-term facilitation on gbaergic synapses in the olfactory bulb. Neuropharmacology, 43:573-583, 2002.

[15] G. Y. Shen, W. R. Chen, J. Mitdgaard, G. M. Shepherd, and M. L. Hines. Computational analysis of action potential initiation in mitral cell soma and dendrites based on dual patch recordings. J. Neurophysiol., 82:3006-3020, 1999.

[16] T. W. Margrie and A. T. Schaefer. Theta oscillation coupled spike latenies yield computational vigour in a mammalian sensory system. J. Physiol, 2002.

[17] A. Papoulis. Probability, random variables, and stochastic processes, second edition. McGraw-Hill Book Co., New York, 1984.

[18] D. Martinez. Oscillatory synchronization requires precise and balanced feedback inhibition in a model of the insect antennal lobe. Neural Computation, In press, 2005.

[19] G. Laurent and H. Davidowitz. Encoding of olfactory information with oscillating neural assemblies. Science, 265:1872-1875, 1994. 